\title{
Article \\ A Cross Sectional Sampling Reveals Novel Coronaviruses in Bat Populations of Georgia
}

\author{
Lela Urushadze ${ }^{1}$, George Babuadze ${ }^{1,2}$, Mang Shi ${ }^{3}$, Luis E. Escobar ${ }^{4}\left(\mathbb{D}\right.$, Matthew R. Mauldin ${ }^{5}$ (D), \\ Ioseb Natradeze ${ }^{6}$, Ann Machablishvili ${ }^{1}$, Tamar Kutateladze ${ }^{1}$, Paata Imnadze 1,7, Yoshinori Nakazawa ${ }^{5}$ \\ and Andres Velasco-Villa ${ }^{5, *(D)}$
}

check for updates

Citation: Urushadze, L.; Babuadze, G.; Shi, M.; Escobar, L.E.; Mauldin, M.R.; Natradeze, I.; Machablishvili,

A.; Kutateladze, T.; Imnadze, P.;

Nakazawa, Y.; et al. A Cross Sectional Sampling Reveals Novel

Coronaviruses in Bat Populations of

Georgia. Viruses 2022, 14, 72.

https://doi.org/10.3390/

v14010072

Academic Editors: Stefania Leopardi, Paola De Benedictis and

Wanda Markotter

Received: 13 November 2021

Accepted: 18 December 2021

Published: 31 December 2021

Publisher's Note: MDPI stays neutral with regard to jurisdictional claims in published maps and institutional affiliations.

Copyright: (C) 2021 by the authors. Licensee MDPI, Basel, Switzerland. This article is an open access article distributed under the terms and conditions of the Creative Commons Attribution (CC BY) license (https:// creativecommons.org/licenses/by/ $4.0 /)$.
1 National Center for Disease Control and Public Health, Tbilisi 0198, Georgia; lelincdc@gmail.com (L.U.); gbabuadze@gmail.com (G.B.); A.Machablishvili@ncdc.ge (A.M.); tamar_kutateladze@yahoo.com (T.K.); pimnadze@ncdc.ge (P.I.)

2 Biological Sciences Platform, Sunnybrook Research Institute, Sunnybrook Health Sciences Centre, Main Campus, University of Toronto, Toronto, ON M4N 3M5, Canada

3 Centre for Infection and Immunity Studies, School of Medicine, Sun Yat-Sen University, Guangzhou 510080, China; shim23@mail.sysu.edu.cn

4 Department of Fish and Wildlife Conservation, Virginia Polytechnic Institute and State University, Blacksburg, VA 24601, USA; escobar1@vt.edu

5 Centers for Disease Control and Prevention, 1600 Clifton Rd. NE, Atlanta, GA 30333, USA; yik5@cdc.gov (M.R.M.); inp7@cdc.gov (Y.N.)

6 Institute of Zoology, Campus S, Ilia State University, Tbilisi 0162, Georgia; ioseb.natradze@iliauni.edu.ge

7 Department of Public Health and Epidemiology, Faculty of Medicine, Main Campus, Ivane Javakhishvili Tbilisi State University, Tbilisi 0179, Georgia

* Correspondence: dly3@cdc.gov

\begin{abstract}
Mammal-associated coronaviruses have a long evolutionary history across global bat populations, which makes them prone to be the most likely ancestral origins of coronavirus-associated epidemics and pandemics globally. Limited coronavirus research has occurred at the junction of Europe and Asia, thereby investigations in Georgia are critical to complete the coronavirus diversity map in the region. We conducted a cross-sectional coronavirus survey in bat populations at eight locations of Georgia, from July to October of 2014. We tested 188 anal swab samples, remains of previous pathogen discovery studies, for the presence of coronaviruses using end-point pancoronavirus RT-PCR assays. Samples positive for a $440 \mathrm{bp}$ amplicon were Sanger sequenced to infer coronavirus subgenus or species through phylogenetic reconstructions. Overall, we found a 24.5\% positive rate, with $10.1 \%$ for Alphacoronavirus and $14.4 \%$ for Betacoronavirus. Albeit R. euryale, R. ferrumequinum, M. blythii and M. emarginatus were found infected with both CoV genera, we could not rule out $\mathrm{CoV}$ co-infection due to limitation of the sequencing method used and sample availability. Based on phylogenetic inferences and genetic distances at nucleotide and amino acid levels, we found one putative new subgenus and three new species of Alphacoronavirus, and two new species of Betacoronavirus.
\end{abstract}

Keywords: coronavirus; bats; georgia; eastern europe; phylogeny; alphacoronavirus; betacoronavirus

\section{Introduction}

Coronavirus (CoV) infection in humans (e.g., 229E, NL63) and other mammals were first reported in the 1960s [1,2]. CoVs are highly pathogenic for livestock, pets, wild animals, as well as birds where they were first described in chickens in the early 1930s [3,4]. During the period 2002-2003, coronaviruses were recognized as zoonotic agents with high pandemic potential after an outbreak of severe acute respiratory syndrome (SARS) that caused 8096 cases and 774 deaths [5,6]. In 2012, the Middle East respiratory syndrome (MERS) emerged as a new public health concern for it spread rapidly to several countries around the globe (mainly the Arabian Peninsula and the Republic of Korea) and for showing 
a considerably higher mortality rate (35\%) than SARS. As of 31 July 2021, MERS has been implicated in 2578 confirmed cases and 888 deaths [6]. The most recent pandemic involving a CoV, SARS-CoV-2 resulted in coronavirus disease 19 (COVID-19), began during 2019 and has caused more than 260 million cases and more than 5 million deaths, confirmed as of 10 December 2021 [7]. The imminent pandemic potential of CoVs has triggered a global hunt for the most likely wildlife reservoir hosts to gain a better understanding of the origins and evolutionary history of CoVs to predict future pandemics [8].

In 2018, the International Committee on Taxonomy of Viruses (ICTV) refined the classification of CoVs to re-organize a greater viral diversity based on newly discovered viruses [6]. Thus, CoVs are within the Riboviria realm that encompasses all viral families having an RNA-dependent RNA polymerase (RdRp), Orthornavirae kingdom, Pisuviricota phylum, Pisoniviricetes class, Nidovirales order, Cornidovirineae suborder, and Coronaviridae family. Coronaviridae was further separated into two subfamilies, Letovirinae primarily affecting amphibians, and Orthocoronavirinae affecting vertebrates. Orthocoronavirinae comprises four genera, Alphaconoravirus, Betacoronavirus, Deltacoronavirus, Gammacoronavirus [9]. Alphacoronaviruses (AlphaCoVs) and betacoronaviruses (BetaCoVs) mainly affect mammals with their greatest health impact in livestock and humans, while deltacoronaviruses (DeltaCoVs) and gammacoronaviruses (GammaCoV) generally affect birds [6,9]. The family Coronaviridae currently recognizes 2 subfamilies, 5 genera, 26 subgenera and 46 species (https:/ / talk.ictvonline.org/taxonomy/ accessed on 1 November 2021).

The reservoir host of SARS-CoV-2 has yet to be confirmed, it has been suggested that there may be cryptic circulation in an unidentified intermediate host species $[5,8]$. Notably, CoV genera of public and animal health concerns share common ancestries with CoVs clades circulating in bats around the world [10]. Phylogenetic reconstructions suggest that bats may have the longest evolutionary history with CoVs justifying an ecological bat-focused investigation screening for $\mathrm{CoVs}$ with pandemic potential $[6,11]$. Although several studies have examined the presence of CoVs in bats throughout Europe and Asia [12,13], there is still very limited information about bat-CoV diversity in the South Caucasus region [14]. Georgia spans over $69,700 \mathrm{~km}^{2}$, with diverse habitats and climates, including coastline to the west, high peaked mountains to the north, rich grasslands to the south, and more arid regions to the southeast, making it an ideal place to sample multiple environments of the South Caucasus.

Given that Georgia is located on the crossroads of Europe, Asia and the Middle East, the country is expected to share bat species and viruses of these three regions $[15,16]$. Over the last decade, Georgia's National Centers for Disease Control has been conducting investigations to search for close relatives of a broad spectrum of pathogens of public health concern potentially circulating in bat populations of this country namely, Leishmania spp., Bartonella spp., Brucella spp., Leptospira spp., Yersinia spp., Lyssavirus spp., and Hantavirus [17-19]. Thus, we conducted a cross-sectional retrospective survey on CoVs in bats across Georgia to fulfill a gap in the understanding of the evolution, ecology and biogeography of CoVs.

\section{Materials and Methods}

\subsection{Sampling}

Bats were collected from July to October 2014 at eight locations within four regions of Georgia (Table 1) to look for close relatives of pathogens causing bacterial, parasitic or viral diseases of public health concern in the region. Animal experiments described in this study were performed in compliance with the Ministry of Environment and Natural Resources Protection of Georgia, special permission \#4001, 18 July 2014, in accordance with Animal Care Ethics Committee at the National Center For Disease Control and Public Health of Georgia. Animal handling techniques followed CDC IACUC protocol \#2096FRAMULX-A3. 
Table 1. Detailed location of collection sites and type of land use or cover. Overall and per genus coronavirus positive rate.

\begin{tabular}{|c|c|c|c|c|c|}
\hline Location & Coordinates Long, Lat/Habitat & Tested & Positive & $\begin{array}{c}\text { Overall CoV Positive Rate } \\
(\%)\end{array}$ & $\begin{array}{c}\text { Positive Rate Per CoV } \\
\text { Genus }\end{array}$ \\
\hline $\begin{array}{l}\text { David Gajeri, } \\
\text { Tetri Senakebi }\end{array}$ & $\begin{array}{cc} & 41.536, \\
& 45.257 \\
\text { Mostly natural vegetation in a mosaic with } \\
\text { cropland }\end{array}$ & 10 & 2 & 20 & $\begin{array}{c}\alpha=10 \% n=1 \\
\beta=10 \% n=1 \\
\text { Neg }=80 \% \\
n=8\end{array}$ \\
\hline $\begin{array}{l}\text { Gardabani Managed } \\
\text { Reserve }\end{array}$ & $\begin{array}{c}41.376, \\
45.079 \\
\text { Mostly natural vegetation in a mosaic with } \\
\text { cropland }\end{array}$ & 49 & 7 & 14 & $\begin{array}{c}\alpha=2 \% n=1 \\
\beta=12 \% n=6 \\
\text { Neg }=86 \% \\
n=43\end{array}$ \\
\hline Tskaltubo, cave Gliana & $\begin{array}{c}42.373 \\
42.597 \\
\text { Mostly cropland in a mosaic with natural } \\
\text { vegetation }\end{array}$ & 92 & 31 & 34 & $\begin{array}{l}\alpha=16.5 \% n=15 \\
\beta=17.5 \% n=16 \\
\quad \mathrm{Neg}=66 \% \\
\quad n=60\end{array}$ \\
\hline Chiatura.Taroklde cave & $\begin{array}{c}42.345 \\
43.308 \\
\text { Rainfed cropland }\end{array}$ & 10 & 2 & 20 & $\begin{array}{c}\alpha=20 \% n=2 \\
\text { Neg }=66.7 \% \\
n=4\end{array}$ \\
\hline $\begin{array}{l}\text { Chkhorotsku. Cave } \\
\text { Lescurcume }\end{array}$ & $\begin{array}{c}42.529 \\
42.102 \\
\text { Mostly cropland in a mosaic with natural } \\
\text { vegetation }\end{array}$ & 17 & 4 & 24 & $\begin{array}{c}\beta=24 \% n=19 \% \\
\mathrm{Neg}=81 \% \\
n=17\end{array}$ \\
\hline Saadamio Senaki & $\begin{array}{c}42.324, \\
42.103 \\
\text { Mostly trees and shrubs in a mosaic of herbaceous } \\
\text { cover }\end{array}$ & 5 & 0 & 0 & $\begin{aligned} \mathrm{Neg} & =100 \% \\
n & =5\end{aligned}$ \\
\hline Tetritskaro, Sabneleti & $\begin{array}{c}41.581 \\
44.582 \\
\text { Rainfed cropland }\end{array}$ & 4 & 0 & 0 & $\begin{aligned} \mathrm{Neg} & =100 \% \\
n & =4\end{aligned}$ \\
\hline Sveri kvabkari & $\begin{array}{c}42.224 \\
43.302 \\
\text { Rainfed cropland }\end{array}$ & 1 & 0 & 0 & $\begin{array}{c}\mathrm{Neg}=100 \% \\
n=1\end{array}$ \\
\hline
\end{tabular}

Natural vegetation $=$ trees, shrubs and herbaceous cover.

Mist nets and hand nets were utilized depending upon roost type (e.g., caves, buildings, attics). The number of individuals caught per roost and per species was authorized with anticipation by the Ministry of Environment and Natural Resources Protection of Georgia. Bats were individually placed in cotton bags to be transported to the laboratory the day after capture.

Bats were euthanized in a BSL2+ laboratory facility at the NCDC-Lugar Center. Specimens, were subsequently, measured (weight and length), sexed, and morphologically inspected for species identification [20]. Rectal and oral swabs, as well as tissues such as brain, liver, lung, spleen and intestines were collected and stored at $-80^{\circ} \mathrm{C}$ for subsequent testing. Rectal swabs were the only specimens available to search for coronaviruses for this retrospective study. All tissues were committed to search for other pathogens such as Leshmania spp., Yersinia spp., Leptospira spp., Bartonella spp., Brucella spp., Lyssavirus spp., Hantavirus [17-19].

\subsection{RNA Extraction and PCR Amplification}

A total of 188 rectal swabs diluted in $1 \mathrm{~mL}$ of virus transport media (VTM) were vortexed and spun down in a refrigerated microcentrifuge for $5 \mathrm{~min}$ at 10,000 rpm. For total RNA extraction, $140 \mu \mathrm{L}$ of the VTM homogenate were placed in a new tube with $560 \mu \mathrm{L}$ of lysis buffer from the QIAamp Viral RNA Mini Kit (Qiagen, Germantown, MD, USA) according to the manufacturer's instructions. All 188 total RNA extracts were screened with a one-step RT-PCR method described previously that uses degenerated primers targeting a relatively conserved $180 \mathrm{bp}$ region within the RNA-dependent RNA polymerase (RdRp) gene [21]. A second $440 \mathrm{bp}$ non-overlapping fragment was amplified as described by Lelli et al. [21], which together with the 180 bp product would generate an approximately $600 \mathrm{bp}$ sequence that can be used for phylogenetic characterization to infer, genus, subgenus and species with greater confidence $[6,9,11,21]$. Amplicons were gel purified using QIAGEN MiniElute ${ }^{\circledR}$ Gel Extraction Kit (250) and subsequently cycle sequenced using the BigDye Terminator Kit, version 3.1 (Applied Biosystems, Foster City, CA, USA) to obtain a consensus sequence. Cycle sequencing products were purified by precipitation with ice-cold $100 \%$ isopropanol once, and then with $70 \%$ isopropanol. Sequencing was conducted using an Applied Biosystems ABI Prism 3130 XL sequencer with data collection software version 4.0. 


\subsection{Sequence Analyses and Phylogenetic Reconstructions}

Excellent quality sequences were obtained in only 9 out of the 46 positive hits $(20 \%)$ for the $180 \mathrm{bp}$ RT-PCR screening amplicon. Thus, consistent concatenation with their $440 \mathrm{bp}$ counterparts to generate a $\sim 600$ bp sequence was not possible. Nonetheless, we were able to obtain high-quality sequences ranging from 385 to $440 \mathrm{bp}$ in all $46 \mathrm{CoV}$ positive samples using the $440 \mathrm{bp}$ RT-PCR amplicon. Thus, we trimmed all 46 sequences to $411 \mathrm{bp}$ that corresponded to the longest sequence length we could obtain with GenBank accession numbers OL791325 to OL791370. This $411 \mathrm{bp}$ fragment was used to search for homolog sequences ranging from 65 to $98 \%$ nucleotide identity, with $100 \%$ query coverage, in the GenBank database using the nucleotide-NCBI-BLAST and MOLEBLAST tools [22]. Gathered GenBank reference sequences were then used to reconstruct preliminary phylogenetic trees for initial genus-level identification (data not shown). Moreover, we used review articles $[6,10,11,23-27]$ to identify $\mathrm{CoV}$ reference sequences representing all currently ICTV recognized subgenera and species pertaining to Alphacoronavirus and Betacoronavirus [9]. We retrieved 164 reference sequences encompassing 14 subgenera and 19 species pertaining to Alphacoronavirus, and 106 reference sequences comprising 5 subgenera and 14 species within Betacoronavirus. All sequences were aligned with the $46 \mathrm{Georgian} \mathrm{CoV}$ positive sequences using MUSCLE [28] and trimmed to $411 \mathrm{bp}$ using BioEdit [29]. Taxa were subsequently divided into two datasets, one for BetaCoV that encompassed $106 \mathrm{BetaCoV}$ reference sequences, $27 \mathrm{CoV}$ sequences obtained from Georgian bats and 5 taxa representing some AlphaCov species, while the second data set comprised $164 \mathrm{AlphaCoV}$ reference sequences, 19 sequences from Georgian bats and $4 \mathrm{BetaCoV}$ taxa encompassing different species. MEGA7 [30], was used to determine the most appropriate model of molecular evolution for these combined datasets. The GTR + G + I substitution model was chosen, out of 24 models tested, for our phylogenetic reconstructions based on the Bayesian information criterion (BIC), Table S1 [30]. A Bayesian phylogenetic reconstruction using MrBayes V 3.2 software [31], was run on these two datasets with two independent runs under the GTR + G + I substitution model with 15 million generations, 4 Markov chains each run and the sampling for tree parameters every 1000 generations to assess branch support and/or calculation of Bayesian posterior probabilities. Using the pairwise alignment tool available in BioEdit [29], we calculated both the average nucleotide identity (ANI) and the average amino acid identity (AAI) by comparing one on one sequences of the Georgian CoV clusters (separately, from both the AlphaCoV and BetaCoV alignments) with taxa of their closest subgenera, species, as well as with unidentified closest $\mathrm{CoVs}$ neighbors observed in the phylogenetic reconstruction. The subgenera and species demarcation criterion suggested in the current literature were also used to assign Georgian $\mathrm{CoV}$ clusters to these taxonomical classification levels $[6,9,11,23,27]$.

To better appreciate the extent of the geographic distribution of AlphaCoV and Beta$\mathrm{CoV}$ found in bat species of Georgia with those bat species harboring highly similar CoVs through Eurasia, we mapped their natural geographic distribution using species range data from the International Union for Conservation of Nature IUCN red list of threatened species [32-44]. Restricting ranges to the geography currently occupied by each species according to expert's records (i.e., extant-resident). Scientific names and synonymies were corroborated following the standards of the Integrated Taxonomic Information System. Bat species were then grouped based on the genus of the coronavirus detected (i.e., AlphaCoV and $\mathrm{BetaCoV})$. To create the maps, administrative boundaries were generated from maps downloaded from DIVA-GIS. Spatial data were handled and displayed using ArcGIS 10.8 (ESRI 2021 and R R Core Team 2021) [45,46].

\section{Results}

Of the 188 bat rectal swabs, $10 \%(19 / 188)$ were positive for AlphaCoV RNA and 14\% (27/188) contained BetaCoV s RNA, with an overall positive rate of $24.5 \%$ (46/188), (Table 1 and Table S2. Please see Supplementary Materials). In regard to gender, we captured 56\% females $(106 / 188)$ and $44 \%$ males $(82 / 188)$, (Table S2). Approximately $29 \%(31 / 106)$ of 
tested females were positive for CoV RNA with 11.3\% (12/106) containing AlphaCoV and $17.9 \%$ (19/106) BetaCoV, (Table S2). Meanwhile, males had a 18.3\% (15/82) positivity rate to CoV RNA, with $8.5 \%$ (7/82) to AlphaCoV and 9.8\% (8/82) to BetaCoV (Table S2).

A total of 183 bats were collected at 7 locations with different degrees of agricultural perturbation across Georgia, where we found a 25\% (46/183) CoV positivity rate (Table 1). The eighth collection site at Saadamio cave, village Saadamio of Senaki municipality, presented an unperturbed landscape dominated by trees, shrubs embedded in a mosaic of herbaceous cover, where we only caught $5 \mathrm{CoV}$ negative individuals (Table 1 ). The overall $\mathrm{CoV}$ positive rate for all eight collection sites was 24.5\% (46/188), (Table 1, Figure 1).

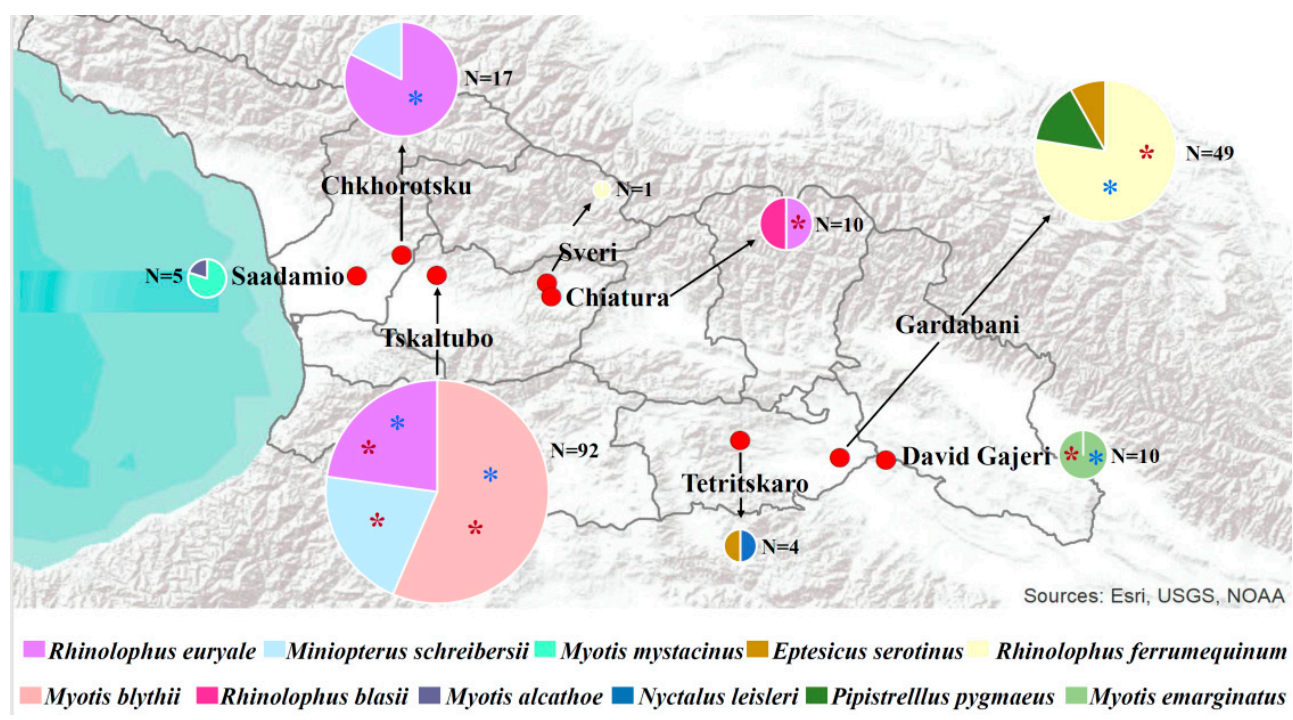

Figure 1. Geographic location and relative proportion of bat species collected per collection site. Red circles indicate the approximate location of collection site. Pie charts indicate the relative proportion of each bat species collected per site. Size of the pie charts indicates the relative number of samples collected per site. Asterisks within the pie indicate whether individuals of that species tested positive for alpha (red) and/or beta CoVs (blue). Lack of asterisks indicates no CoV were detected in a bat species and/or site. Color codes for each bat species represented in the pie charts are indicated in the lower part of the figure.

Collected bats belong to two families Rhinolophidae $(n=84)$ that encompassed a single genus with three species namely, Rhinolophus euryale $(n=40)$, R. ferrumequinum $(n=39)$ and R. blasii $(n=5)$, and Vespertilionidae $(n=104)$ that comprised 5 genera with a total of 8 species (Table 2). The overall CoV positivity rates within the Rhinolophidae and Vespertilionidae were $25 \%$ and $24 \%$, respectively (Table 2 ). However, Rhinolophus spp. presented higher CoV positive rates for BetaCoV (19\%) than for AlphaCoV (6\%) (with exception of Rhinolophus blasii that was negative for both $\mathrm{CoV}$ genera). In contrast, Miniopterus schreibersii exclusively presented AlphaCoV, while Myotis blythii (35\% overall positivity rate, with $15 \%$ AlphaCoV and $19 \%$ BetaCoV) presented similar positivity rates to those observed in Rhinolophidae bats (Table 2, Figures 2 and 3). The remaining Vespertilionidae species (Eptesicus serotinus, Pipistrellus pygmaeus, Myotis mystacinus, M. alcathoe and Nyctalus leisleri) were CoV negative along this transversal sampling (Table 2, Figure 1). Notably, CoVs were not detected in all bat species in which less than 10 individuals were collected. Albeit different individuals of the same species were found infected with AlphaCoV and BetaCoV within the same locations (Figure 1, Table 2), the sequencing method approach used could not rule out the potential presence of $\mathrm{CoV}$ co-infection among individuals. Geographically, the majority of $\mathrm{CoV}$ positive bats were collected in the Western part of Georgia in the regions of Imereti $(32 / 46=71 \%)$ and Samegrelo $(4 / 46=8.7 \%)$. The remaining 10 positive bats were collected in South-Eastern part of the Country including Kvemo Kartli $(7 / 46=15.2 \%)$. Remarkably, 
only $0.5 \%(1 / 45)$ of $\mathrm{CoV}$ positive bats were collected in the Kakheti region that represents the Eastern part of Georgia (Figure 1, Table S2).

Table 2. Overall and per genus coronavirus positive rate across bat species collected.

\begin{tabular}{|c|c|c|c|}
\hline Species & Tested & Overall CoV Positive Rate (\%) & Positive Rate Per CoV Genus \\
\hline Rhinolophus euryale & 40 & $14 / 40=35$ & $\begin{array}{c}\alpha=4 / 40=10 \\
\beta=10 / 40=25 \\
\text { Negative }=26 / 40=65\end{array}$ \\
\hline Rhinolophus ferrumequinum & 39 & $7 / 39=18$ & $\begin{array}{c}\alpha=1 / 39=3 \\
\beta=6 / 39=15 \\
\text { Negative }=32 / 39=82\end{array}$ \\
\hline Rhinolopus blasii & 5 & 0 & Negative $=5 / 5=100$ \\
\hline Eptesicus serotinus & 6 & 0 & Negative $=6 / 6=100$ \\
\hline Miniopterus schreibersii & 22 & $5 / 22=23$ & $\begin{array}{c}\alpha=5 / 22=23 \\
\text { Negative }=17 / 22=77\end{array}$ \\
\hline Myotis blythii & 52 & $18 / 52=35$ & $\begin{array}{c}\alpha=8 / 52=15 \\
\beta=10 / 52=19 \\
\text { Negative }=34 / 52=66\end{array}$ \\
\hline Myotis emarginatus & 10 & $2 / 10=20$ & $\begin{array}{c}\alpha=1 / 10=10 \\
\beta=1 / 10=10 \\
\text { Negative }=8 / 10=80\end{array}$ \\
\hline Pipistrellus pygmaeus & 7 & 0 & Negative $=7 / 7=100$ \\
\hline Myotis mystacinus & 4 & 0 & Negative $=4 / 4=100$ \\
\hline Nyctalus leisleri & 2 & 0 & Negative $=2 / 2=100$ \\
\hline Myotis alcathoe & 1 & 0 & Negative $=1 / 1=100$ \\
\hline
\end{tabular}

The 19 Georgian AlphaCoVs grouped as two unclassified subgenera ( $n=12$ taxa), and as two unclassified species within two subgenera $(n=7)$, (Figure 2$)$. The largest cluster encompassing 10 sequences (locate at the top of the tree, marked with a bar in color dark red) collected from cave Gliana, village Kumistavi of Tskaltubo municipality corresponded to Georgian bats of three species, Myotis blythii $(n=7)$, Miniopterus schreibersii $(n=2)$, and Rhinolophus euryale $(n=1)$. This cluster formed a monophyletic clade with unclassified $\mathrm{CoV}$ sequences found in Myotis myotis bats from Hungary, Germany, Spain and Italy presenting ANI and AAI values of $97.1 \%$ and 99.5\%, respectively (Figure 2, and Table S2). Other CoVs sequences found in a Myotis daubentonii bat from China, an unidentified bat from Korea and a Myotis sp. from Hong Kong were also identified as closely related to this cluster of Georgia sequences. However, they presented lower overall ANI and AAI values around $87.1 \%$ and $96.1 \%$, respectively. At a more ancestral node (0.94 posterior probability) this cluster of unclassified AlphaCoV, mainly associated with vespertilionid bats from Europe and Asia shared a common ancestry with Pedacovirus I, Pedacovirus and Colacovirus subgenera with more distant overall ANI and AAI values around 77.6\% and 86.6\% (Figure 2).

Two sequences (CoV153 and CoV152) obtained from Rhinolophus euryale bats from cave Taroklde, village Zodi of Chiatura municipality, grouped monophyletically with high support with bat CoV circulating in Rhinolophus blasii from Bulgaria (97.6\% ANI and 100\% AAI) and Rhinolophus ferrumequinum from France (87.1\% ANI and 99.3\% AAI) and Italy (86.4\% ANI and 99.3\% AAI) (Figure 2, Table S2).

We identified a Georgian CoV sequence (GE_CoV10) obtained from a Myotis emarginatus bat as sister taxon of Rhinolophus ferrumequinum alphacoronavirus HuB-2013 (with an ANI of 94.7 and an AAI of 100\%), which falls within the Decacovirus clade (Figure 2). 


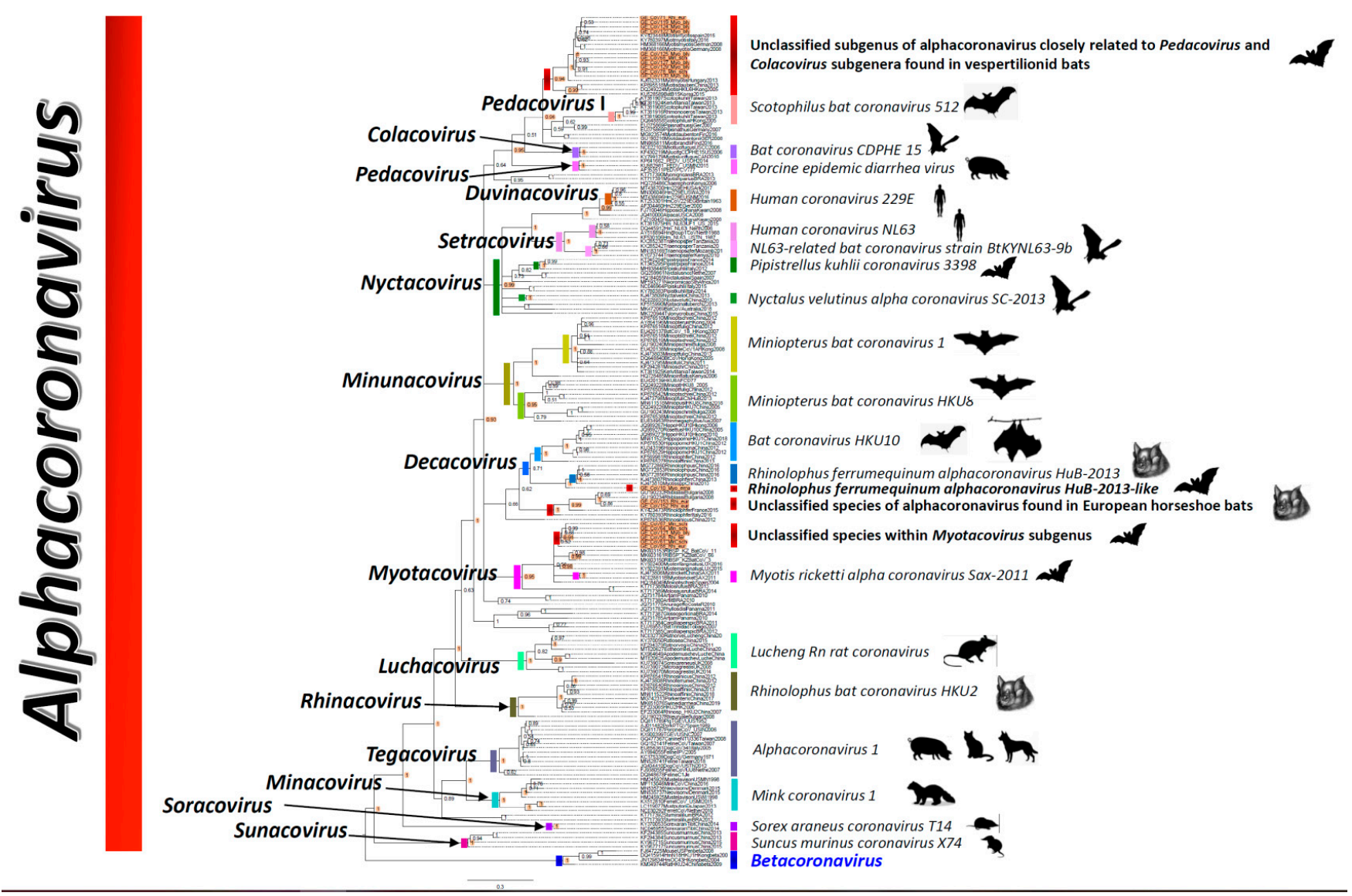

Figure 2. Unrooted Bayesian phylogenetic tree for AlphaCoVs generated with a partial informative $411 \mathrm{bp}$ fragment of the RdRp. Representative BetaCoV taxa were used in the alignment to demonstrate consistent segregation between both genera. Values at nodes represent branch support values expressed as Bayesian posterior probabilities. Scale bar indicates branch lengths. Highlighted nodes are robustly supported with colored bars indicating relevant branches encompassing taxa pertaining to a given subgenus on the left. Bars on the right indicate taxa pertaining to recognized coronavirus species according to the most recent ICTV classification scheme. Taxa highlighted in orange on the right side of the tree indicate coronavirus sequences obtained from Georgian bats. Dark red bars indicate unclassified coronavirus subgenera or species identified herein. Taxa names for all reference sequences in the tree start with their respective GenBank accession number. Animal silhouettes on the extreme right indicate the animal species from which reference sequences were recovered. 
The fourth AlphaCo cluster (comprising 6 sequences) was the most diverse considering the number of bats species it contained, Miniopterus schreibersii $(n=3)$ from cave Gliana at village Kumistavi of Tskaltubo municipality, Rhinolophus ferrumequinum $(n=1)$ from the managed reserve at Gardabani, $R$ euryale $(n=1)$ from cave Gliana at village Kumistavi of Tskaltubo municipality, and Myotis blythii $(n=1)$ from cave Gliana at Tshaltubo. This Georgia cluster was monophyletic with sequences pertaining to Myotis ricketti alpha coronavirus Sax-2011 and Miniopterus bat coronavirus HKU8 bat CoV, as well as with two CoV obtained from Molossus rufus bats from Brazil, all occupying the Myotacovirus clade (Table S1, Figure 2). The closest relatives (albeit they grouped inside a polytomy with a low support value 0.57 ) to this cluster of Georgian bat CoVs were sequences obtained from Myothis blythii from Kazakhstan (91\% ANI and 100\% AAI), Miniopterus schreibersii (90\% ANI and 99.3\% AAI) from Luxemburg and Spain, and Myotis emarginatus (89.8\% ANI and $97.8 \% \mathrm{AAI})$. These Georgian sequences, together with all other sequences inside the polytomy shared a common ancestor with $\mathrm{CoV}$ sequences obtained from Molossus rufus from Brazil (also pertaining to Myotacovirus), with ANI (77.8\%) and AAI (83.2\%) values observed (Figure 2).

Regarding the Georgian bat BetaCoV s, twenty-four sequences obtained from, Rhinolophus euryale $(n=10)$, R. ferrumequinum $(n=6)$, Myotis blythii $(n=7)$, M. emarginatus $(n=1)$ collected from David Gajeri $(n=1)$, Gardabani $(n=6)$, Tskaltubo $(n=13)$, and Chkhorotsku $(n=4)$ segregated within this genus and shared a common ancestor with CoV reference sequences pertaining to the Sarbecovirus and the Severe acute respiratory syndrome-related coronavirus. Interestingly, this group of Georgian bat CoVs presented ANI and AAI values of $96.9 \%$ and $97.81 \%$ with CoVs mainly found in $R$. blasii, $R$. ferrumequinum, and $R$. hipossideros from Bulgaria, Spain, France, Italy and Luxemburg. SARSCoV and SARSCoV-2 viruses obtained from Rhinolophus bats, civets and humans presented ANI values with this group of Georgian BetaCoV s of 88.6\% (97.45\% AAI) and 88.8\% (95.62\% AAI), respectively (Table S2).

Similarly, we found three CoVs obtained from Myotis blythii collected at Tskaltubo that presented the highest average nucleotide and amino acid identities with a group of unclassified merbecoviruses obtained from Vespertilionid bats from Italy (Eptesicus serotinus and Nyctalus noctula 96\% ANI and 97.8\% AAI), China (Myotis pequinius $94.6 \%$ ANI, 95.6\% AAI), and Finland (Eptesicus nilssonii 95.6\% ANI and 97.8\% AAI). This group of unclassified CoVs shared a common ancestry with the 4 species of the Merbecovirus (Figure 3). These three Georgian CoVs had the highest ANI (84.9\%) and AAI (96\%) with the Middle East respiratory syndrome-related coronavirus (Table S2), which then decreased for the remaining three species in the subgenus as follows, Pipistrellus bat coronavirus HKU5 (82\% ANI, 90.5\% AAI), Hedgehog coronavirus 1 (81.3\% ANI, 88.3\% AAI), Tylonycteris bat conoravirus HKU4 (78.8\% ANI, 89\% AAI).

All CoV positive bat species that were found in Georgia presented an overlapping geographic distribution across most of southern Europe, a portion of North Africa and across central Asia. Only the distributions of Myotis blythii and Rhinolophus ferrumequinum's extended to parts of Southeast Asia with the latter having the widest distribution in the region (Figure 4). Myotis ricketti (renamed as Myotis pilosus) whose natural distribution seems to be restricted to Southeast Asia appears to be the primary host of Myotis ricketti alpha coronavirus Sax-2011. However, close relatives of this AlphaCoV species were found circulating in Georgia in Miniopterus schreibersii, Rhinolophus ferrumequinum and Myotis blythii, suggesting that broadly distributed bats species such as Rhinolophus ferrumequinum and Myotis blythii could have dispersed this CoV species to Central Asia and Western Europe (Figure 4). 


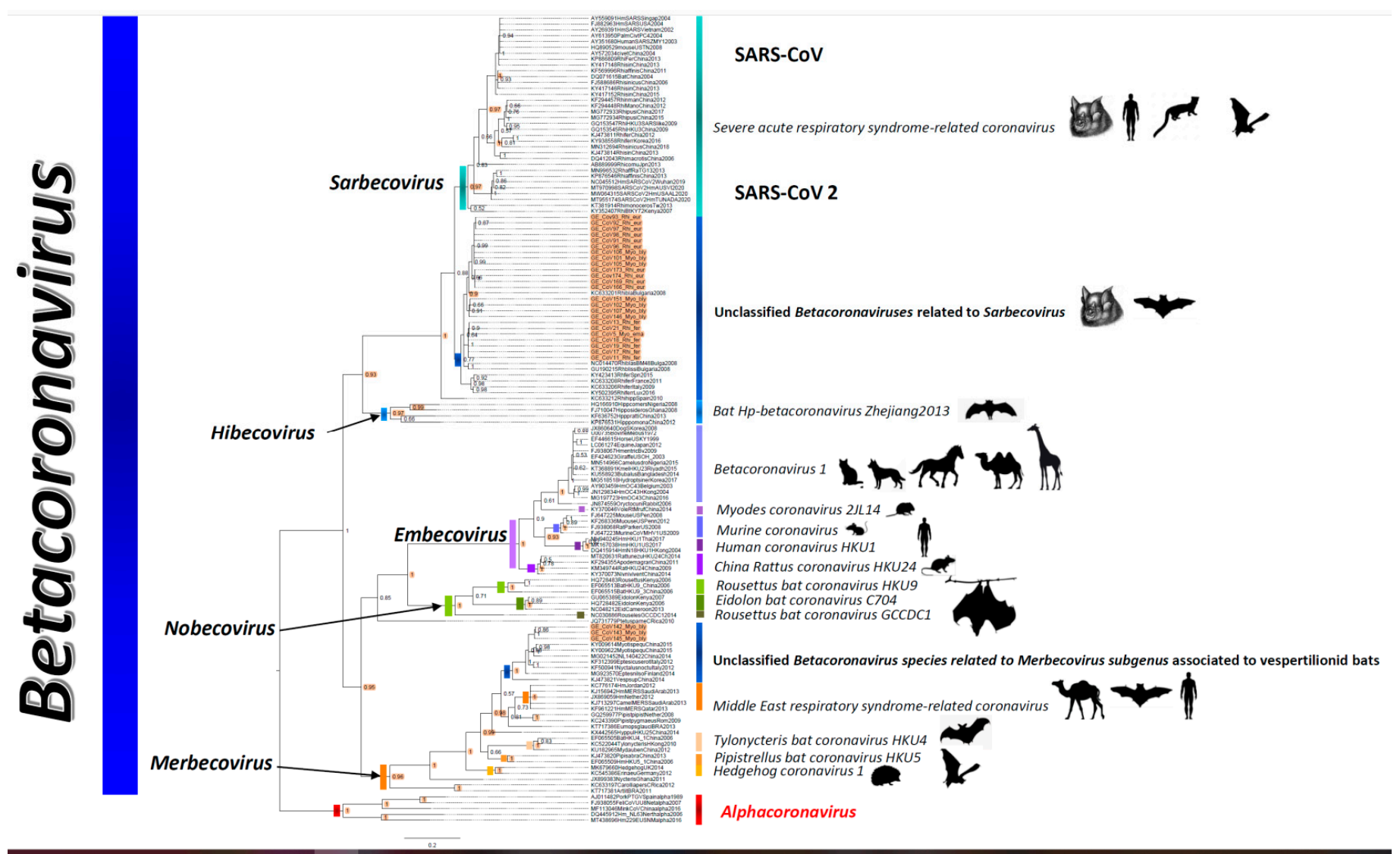

Figure 3. Unrooted Bayesian phylogenetic tree for beta CoVs generated with a partial informative $411 \mathrm{bp}$ fragment of the RdRp. Representative BetaCoV taxa were used in the alignment to demonstrate consistent segregation between both genera. Values at nodes represent branch support values expressed as Bayesian posterior probabilities. Scale bar indicates branch lengths. Highlighted nodes are robustly supported with colored bars indicating relevant branches encompassing taxa pertaining to a given subgenus on the left. Bars on the right indicate taxa pertaining to recognized CoV species according to the most recent ICTV classification scheme. Taxa highlighted in orange on the right side of the tree indicate $\mathrm{CoV}$ sequences obtained from Georgian bats, and the dark blue bars indicate unclassified $\mathrm{CoV}$ species. Taxa names for all reference sequences in the tree start with their respective GenBank accession number. Animal silhouettes on the extreme right indicate the animal species from which reference sequences were recovered. 

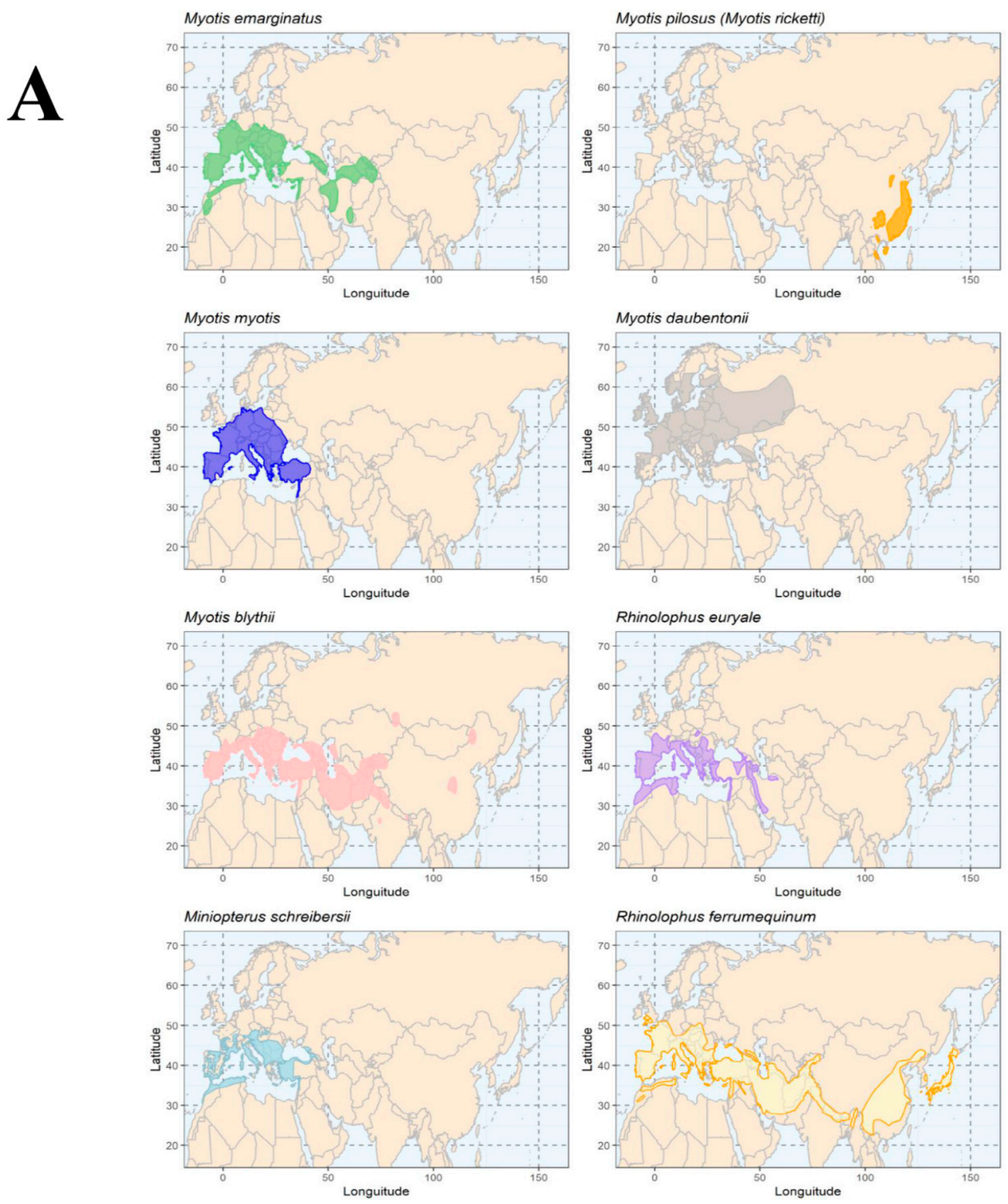

Figure 4. Cont. 

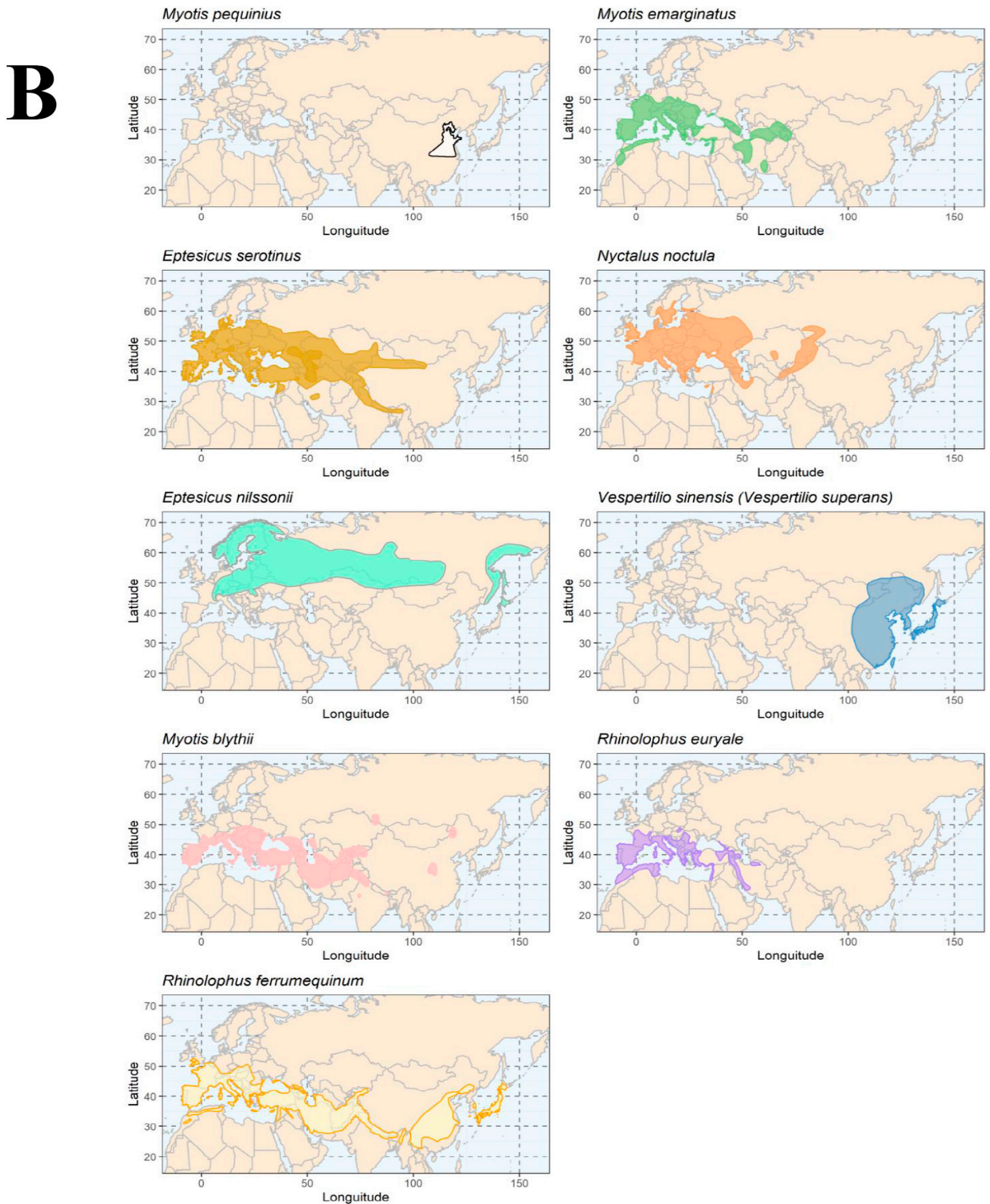

Figure 4. Distribution maps for bat species found to be $\mathrm{CoV}$ positive in this study compared with the geographic range of other bat species presenting highly similar CoVs across Eurasia as observed in our phylogenetic reconstructions. (A) depicts the geographic range of bat species with highly similar AlphaCoV obtained from Georgia with those reported in other countries across Eurasia. (B) depicts the geographic range of bat species with highly similar BetaCoV obtained from Georgia with those reported in other countries across Eurasia. 


\section{Discussion}

The genetic characterization of mammal-associated $\mathrm{CoV}$ using a region spanning $411 \mathrm{bp}$ within the RdRp was robust enough to identify novel CoVs circulating in bat populations across Georgia, albeit no reliable evolutionary relationships among subgenera and species could be established consistent with previous investigations using RdRp fragments shorter than 806 nucleotides $[6,9,23,27]$. Overall, our analyses based on phylogenetic inference, as well as considering the ANI and AAI criteria proposed recently $[6,9,27]$, demonstrated the circulation of one putative unclassified subgenus closely related to the Pedacovirus holotypes and the Colacovirus subgenus. Three unclassified species two of which are within the Decacovirus, and the other one within the Myotacovirus. Additionally, we found two putative unclassified species within Sarbecovirus and Merbecovirus.

Wilkinson et al.; provided robust evidence that strong monophyletic groups demarcating a subgenus and species should have Bayesian posterior probabilities higher than 0.9 [6]. The genera level trees constructed for this investigation depicted with high support values all ICTV recognized subgenera and species and were consistent with previous investigations where robust analyses were conducted $[6,9,11,27]$. Interestingly, most of our Georgian $\mathrm{CoV}$ clades grouped consistently within $\mathrm{CoVs}$ associated with the same bat species or genus across Europe, Asia, Africa or the Americas, which supports the hypothesis that $\mathrm{CoVs}$ have strong epizootiological associations with their bat hosts spanning most of their natural geographic distributions [9,16,23,47-52]. In some instances, there were multiple bat species with the same viruses such as in the SARS-like clade where Rhinolophus spp. and Myotis blythii shared closely related viruses [11,51,53]. Similarly, two different clusters within AlphaCoVs, one that was embedded within Myotacovirus clade from Georgia was dominated by sequences obtained from Miniopterus schreibersii and Rhinolophus spp. However, this group contained a $\mathrm{CoV}$ species mainly associated with Myotis ricketti, while highly similar CoV sequences have been obtained from Myotis blythii in Kazakhstan and Italy and other parts of Europe, suggesting Myotis spp. could be the main reservoir hosts for this CoV [49,53-55]. Another Georgia clade predominantly containing sequences from Myotis blythii, may represent a yet unclassified Myotis blythii alphacoronavirus subgenus, which was found also infecting Rhinolophus euryale and Miniopterus schreibersii bats. Interestingly, the cross-sectional sampling we undertook could not identify an infection pattern among sedentary (Rhinolophus ferrumequinum, R. euryale, R. blasii, Myotis emarginatus, M. alcathoe and Eptesicus serotinus), sedentary or short-distance migrants (M. blythii, M. mystacinus), short-distance seasonal migrant (Miniopterus schreibersii), partially migrant (Pipistrellus pygmaeus) and long-distance migrant (Nyctalus leisleri) bat species, that would intuitively render seasonal and long-distance migrant bat species with a greater diversity of CoVs $[39,40]$. Although Miniopterus schreibersii bats have been found infected either with alpha or beta CoVs throughout Europe, they are predominantly infected with AlphaCoV in South East Asia $[47,51,56]$, as we noted in Georgia. All together, these results indicate relatively frequent $\mathrm{CoV}$ spillover infections among bat species sharing roosts highlighting a broad susceptibility of multiple bats species to $\mathrm{CoV}$ infection that could potentially lead to host shifts [23,27]. Alternatively, these results may suggest that there might be multiple bat hosts species associated with the circulation maintenance of each viral genus acting as host-community complexes. Bat species with broad natural ranges could be playing a central role in the gradual dissemination of alpha and beta CoVs to other susceptible bats species across Eurasia, making $\mathrm{CoV}$ infection persistence and its dissemination dynamics a rather complex issue across bat populations of World.

Wilkinson and colleagues proposed an ANI threshold of $77.6 \%$ for different subgenera within alpha CoVs and 71.7 for BetaCoVs with high confidence levels [6]. Conversely, Geldenhuys and colleagues report pairwise amino acid average divergence thresholds (that we converted to identity values using the following formula, 100-divergence value $=$ identity) that demarcate species to subgenus with AAI values lower than $92.4 \%$ and from subgenus to genus with AAI values lower than 85.3\% [27]. Thereby, all our 
inferences to characterize Georgian $\mathrm{CoV}$ to subgenus and species levels fell into expected ranges $[6,27]$.

Herein, we report for the first time the co-circulation of alpha and BetaCoVs in bat species belonging to Vespertilionidae and Rhinolophidae across Georgia, which constitutes the first report for the region [14]. Mammal-associated $\mathrm{CoV}$ seem ubiquitous in populations of the Vespertilionidae and Rhinolophidae bat families across the world [47-50,55]. Nonetheless, there are few countries within the Americas, Europe, Asia, Africa and Australia where both $\mathrm{CoV}$ genera co-circulate in native bat populations [47-55,57-63]. Likely, countries with co-circulation of both mammal-associated $\mathrm{CoV}$ genera, reflect an intensified surveillance in their bat populations, particularly after $\mathrm{CoVs}$ found in both bat families were predominantly associated with human acute respiratory syndrome epidemics and pandemics in the recent two decades $[5,25,26,64]$.

Our results are not surprising since Georgia contains fauna native to both Europe and Asia and is relatively close to the Middle East [15]. Its geographic location at the middle of this great bio-diversity corridor, explains the outstanding diversity of $\mathrm{CoV}$ found, despite its relatively small territory $\left(69,700 \mathrm{~km}^{2}\right)$, as well as predicts a similar CoV richness for all the unexplored countries in the surrounding region [16]. We noticed that all Georgian bat species found positive with $\mathrm{CoVs}$ had broad natural geographic distributions across Eurasia, parts of Africa and the Americas. Strikingly, Georgian CoVs share recent common ancestry with $\mathrm{CoV}$ found circulating in other widely distributed bats species across Eurasia and the Americas. This empirical observation suggests mammal-associated CoVs may undergo complex transmission dynamics across sympatric bat communities, perhaps highlighting the susceptibility of multiple bat species to $\mathrm{CoV}$ infection. Recent modeling efforts have assessed the risk for the presence of bat-associated zoonoses globally [56]. Some of these results suggest Western Asia (especially the region comprising Georgia) is a hot spot for the presence of BetaCoV with pandemic potential, outside of South East Asia Western Europe and Central Asia [15,16].

The great $\mathrm{CoV}$ diversity found in single caves and the close proximity among positive locations suggests $\mathrm{CoV}$ co-infections are likely to occur in bat populations across the region. However, the sequencing approach we used and the amount of sample available hampered the detection of $\mathrm{CoV}$ co-infections in our sample set. In addition, the poor sequencing results obtained in the $180 \mathrm{bp}$ pan-coronavirus RT-PCR could be due to a poor amplicon yield associated to low primer affinity (due to more than two sequence mismatches) along the highly variable region of the RdRp gene this 180 bp RT-PCR targets. This is clearly indicated by the 7 degenerated positions in the primer set used to amplify this $180 \mathrm{bp}$ region [21]. Thus, this short $180 \mathrm{bp}$ amplicon is practically useless for further $\mathrm{CoV}$ phylogenetic characterization. For future research we would only recommend the use of the $440 \mathrm{bp}$ long amplicon (that anneals along a much more conserved region within the RdRp), which could be used for screening and phylogenetic characterization when a limited amount of sample is available [21]. Moreover, the sequencing of such an amplicon by next generation platforms may allow the detection of $\mathrm{CoV}$ co-infections in single bats.

\section{Conclusions}

CoVs found in Georgian bat populations were strikingly diverse. Some of these Georgian CoVs were closely related to those that caused human pandemics with epicenters in China (SARS) and the Middle East (MERS). We predominantly described new bat $\mathrm{CoV}$ species within previously described subgenera within Alphacoronavirus and Betacoronavirus, with one likely novel, yet unclassified, a subgenus of Alphacoronavirus associated with Myotis blythii. Our results provide further insight into the global genetic structure of $\mathrm{CoV}$ associated with bats in Eurasia and may inform the overall regional CoV diversity, critical to assess the global epidemiology of emerging diseases. 
Supplementary Materials: The following are available online at https: / www.mdpi.com/article / 10.3390/v14010072/s1, Tables: Bat species captured and associated coronaviruses in the present study. The GTR + G + I substitution modeling and phylogenetic recon-structions based on the Bayesian information criterion (BIC). Tables S1-S4: Shows all the metadata associated to each of the samples collected in Georgia, which include collection date, location's name, sample number, identified bat species, taxon's name, morphometric characteristics corresponding to each bat collected. Putative CoV genus identified according to the phylogenetic inference, BLAST best match and overall nucleotide identity over a $411 \mathrm{bp}$ fragment. Red color rows indicate taxa identified as positive for alphacoronavirus RNA and blue color rows indicate taxa identified as positive for beta CoV RNA via RT-PCR.

Author Contributions: Conceptualization, L.U. and A.V.-V.; methodology, A.V.-V., G.B., L.U., L.E.E., A.M., T.K., P.I. and M.S.; formal analysis, A.V.-V., L.E.E., M.S., M.R.M., G.B., I.N. and L.U.; investigation, L.U., G.B., I.N., A.M., T.K., P.I. and A.V.-V.; resources, L.U., A.V.-V.; data curation, L.U., G.B., I.N., P.I., M.R.M., L.E.E., M.S. and A.V.-V.; writing, A.V.-V., G.B., L.U., M.R.M., L.E.E., M.S. and L.E.E.—original draft preparation, A.V.-V., G.B., L.U. and M.R.M.; writing-review and editing, A.V.-V., G.B., L.U., M.R.M., L.E.E., M.S. and Y.N.; visualization, A.V.-V., L.E.E. and M.S.; supervision, A.V.-V. and L.U.; project administration, L.U. and A.V.-V.; funding acquisition, L.U. and A.V.-V. All authors have read and agreed to the published version of the manuscript.

Funding: This research was funded by The International Science and Technology Center (ISTC) [G-2101] and The APC was funded by US Centers for Disease Control and Prevention (CDC).

Institutional Review Board Statement: Animal experiments described in this study were performed in compliance with the Ministry of Environment and Natural Resources Protection of Georgia, special permission \#4001, 18 July 2014, in accordance with Animal Care Ethics Committee at the National Center For Disease Control and Public Health of Georgia. Animal handling techniques followed CDC IACUC protocol \#2096FRAMULX-A3.

Informed Consent Statement: No human subjects or neither human samples were involved and/or used in this study.

Data Availability Statement: The data that have been used in this study will be available from the corresponding author upon requests.

Acknowledgments: Authors thank Mariana Castaneda-Guzman for her valuable contributions on data handling and visualization.

Conflicts of Interest: The authors declare no conflict of interest. The opinions and conclusions expressed in this report are those of the authors and do not necessarily represent the official position of the CDC or the US Department of Health and Human Services.

\section{References}

1. Hamre, D.; Procknow, J.J. A new virus isolated from the human respiratory tract. Proc. Soc. Exp. Biol. Med. 1966, 121, 190-193. [CrossRef] [PubMed]

2. McIntosh, K.; Dees, J.H.; Becker, W.B.; Kapikian, A.Z.; Chanock, R.M. Recovery in tracheal organ cultures of novel viruses from patients with respiratory disease. Proc. Natl. Acad. Sci. USA 1967, 57, 933-940. [CrossRef] [PubMed]

3. Schalk, A.F. An apparently new respiratory disease of baby chicks. J. Am. Vet. Res. Assoc. 1931, 78, 413.

4. Kahn, J.S.; McIntosh, K. History and recent advances in coronavirus discovery. Pediatr. Infect. Dis. J. 2005, 24, S223-S227. [CrossRef] [PubMed]

5. Souilmi, Y.; Lauterbur, M.E.; Tobler, R.; Huber, C.D.; Johar, A.S.; Moradi, S.V.; Johnston, W.A.; Krogan, N.J.; Alexandrov, K.; Enard, D. An ancient viral epidemic involving host coronavirus interacting genes more than 20,000 years ago in East Asia. Curr. Biol. 2021, 31, 3504-3514.e9. [CrossRef]

6. Wilkinson, D.A.; Joffrin, L.; Lebarbenchon, C.; Mavingui, P. Analysis of partial sequences of the RNA-dependent RNA polymerase gene as a tool for genus and subgenus classification of coronaviruses. J. Gen. Virol. 2020, 101, 1261-1269. [CrossRef]

7. WHO. Coronavirus (COVID-19) Dashboard. Available online: https:/ / covid19.who.int/ (accessed on 10 December 2021).

8. Mallapaty, S. The search for animals harbouring coronavirus-And why it matters. Nature 2021, 591, 26-28. [CrossRef]

9. Coronaviridae Study Group of the International Committee on Taxonomy of Viruses. The species Severe acute respiratory syndrome-related coronavirus: Classifying 2019-nCoV and naming it SARS-CoV-2. Nat. Microbiol. 2020, 5, 536-544. [CrossRef] [PubMed]

10. Wong, A.C.P.; Li, X.; Lau, S.K.P.; Woo, P.C.Y. Global Epidemiology of Bat Coronaviruses. Viruses 2019, 11, 174. [CrossRef] 
11. Drexler, J.F.; Gloza-Rausch, F.; Glende, J.; Corman, V.M.; Muth, D.; Goettsche, M.; Seebens, A.; Niedrig, M.; Pfefferle, S.; Yordanov, S.; et al. Genomic characterization of severe acute respiratory syndrome-related coronavirus in European bats and classification of coronaviruses based on partial RNA-dependent RNA polymerase gene sequences. J. Virol. 2010, 84, 11336-11349. [CrossRef]

12. Gloza-Rausch, F.; Ipsen, A.; Seebens, A.; Göttsche, M.; Panning, M.; Drexler, J.F.; Petersen, N.; Annan, A.; Grywna, K.; Müller, M.; et al. Detection and prevalence patterns of group I coronaviruses in bats, northern Germany. Emerg. Infect. Dis. 2008, 14, 626-631. [CrossRef]

13. Memish, Z.A.; Alhakeem, R.; Stephens, G.M. Saudi Arabia and the emergence of a novel coronavirus. East. Mediterr. Health J. 2013, 19 (Suppl. 1), S7-S11. [CrossRef] [PubMed]

14. Phelps, K.L.; Hamel, L.; Alhmoud, N.; Ali, S.; Bilgin, R.; Sidamonidze, K.; Urushadze, L.; Karesh, W.; Olival, K.J. Bat Research Networks and Viral Surveillance: Gaps and Opportunities in Western Asia. Viruses 2019, 11, 240. [CrossRef]

15. Allen, T.; Murray, K.A.; Zambrana-Torrelio, C.; Morse, S.S.; Rondinini, C.; Di Marco, M.; Breit, N.; Olival, K.J.; Daszak, P. Global hotspots and correlates of emerging zoonotic diseases. Nat. Commun. 2017, 8, 1124. [CrossRef]

16. Anthony, S.J.; Johnson, C.K.; Greig, D.J.; Kramer, S.; Che, X.; Wells, H.; Hicks, A.L.; Joly, D.O.; Wolfe, N.D.; Daszak, P.; et al. Global patterns in coronavirus diversity. Virus. Evol. 2017, 3, vex012. [CrossRef]

17. Gu, S.H.; Lim, B.K.; Kadjo, B.; Arai, S.; Kim, J.A.; Nicolas, V.; Lalis, A.; Denys, C.; Cook, J.A.; Dominguez, S.R.; et al. Molecular phylogeny of hantaviruses harbored by insectivorous bats in Côte d'Ivoire and Vietnam. Viruses 2014, 6, 1897-1910. [CrossRef]

18. Bai, Y.; Urushadze, L.; Osikowicz, L.; McKee, C.; Kuzmin, I.; Kandaurov, A.; Babuadze, G.; Natradze, I.; Imnadze, P.; Kosoy, M. Molecular Survey of Bacterial Zoonotic Agents in Bats from the Country of Georgia (Caucasus). PLoS ONE. 2017, 12, e0171175. [CrossRef]

19. Urushadze, L.; Bai, Y.; Osikowicz, L.; McKee, C.; Sidamonidze, K.; Putkaradze, D.; Imnadze, P.; Kandaurov, A.; Kuzmin, I.; Kosoy, M. Prevalence, diversity, and host associations of Bartonella strains in bats from Georgia (Caucasus). PLoS Negl. Trop. Dis. 2017, 11, e0005428. [CrossRef]

20. Dietz, C.; Helversen, O. Identification Key to the Bats of Europe, Version 1.0; Electronical Publication, University of Tübingen: Tübingen, Germany, 2012; p. 72.

21. Lelli, D.; Papetti, A.; Sabelli, C.; Rosti, E.; Moreno, A.; Boniotti, M.B. Detection of coronaviruses in bats of various species in Italy. Viruses 2013, 5, 2679-2689. [CrossRef] [PubMed]

22. Altschul, S.F.; Gish, W.; Miller, W.; Myers, E.W.; Lipman, D.J. Basic local alignment search tool. J. Mol. Biol. 1990, $215,403-411$. [CrossRef]

23. Drexler, J.F.; Corman, V.M.; Drosten, C. Ecology, evolution and classification of bat coronaviruses in the aftermath of SARS. J. Antiviral. Res. 2014, 101, 45-56. [CrossRef]

24. Wassenaar, T.M.; Zou, Y. 2019_nCoV/SARS-CoV-2: Rapid classification of betacoronaviruses and identification of Traditional Chinese Medicine as potential origin of zoonotic coronaviruses. Lett. Appl. Microbiol. 2020, 70, 342-348. [CrossRef]

25. Wu, F.; Zhao, S.; Yu, B.; Chen, Y.M.; Wang, W.; Song, Z.G.; Hu, Y.; Tao, Z.W.; Tian, J.H.; Pei, Y.Y.; et al. A new coronavirus associated with human respiratory disease in China. Nature 2020, 579, 265-269. [CrossRef]

26. Cui, J.; Li, F.; Shi, Z.L. Origin and evolution of pathogenic coronaviruses. Nat. Rev. Microbiol. 2019, 17, 181-192. [CrossRef]

27. Geldenhuys, M.; Mortlock, M.; Epstein, J.H.; Pawęska, J.T.; Weyer, J.; Markotter, W. Overview of Bat and Wildlife Coronavirus Surveillance in Africa: A Framework for Global Investigations. Viruses 2021, 13, 936. [CrossRef] [PubMed]

28. Edgar, R.C. MUSCLE: A multiple sequence alignment method with reduced time and space complexity. BMC Bioinform. 2004, 5, 113. [CrossRef] [PubMed]

29. Hall, T.A. BioEdit: A user-friendly biological sequence alignment editor and analysis program for Windows 95/98/NT. Nucl. Acids Symp. 1999, 41, 95-98. [CrossRef]

30. Kumar, S.; Stecher, G.; Tamura, K. MEGA7: Molecular evolutionary genetics analysis version 7.0 for bigger datasets. Mol. Biol. Evol. 2016, 33, 1870-1874. [CrossRef] [PubMed]

31. Huelsenbeck, J.P.; Ronquist, F. MRBAYES: Bayesian inference of phylogenetic trees. Bioinformatics 2001, 17, 754-755. [CrossRef]

32. Kruskop, S.V.; Godlevska, L.; Bücs, S.; Çoraman, E.; Gazaryan, S. Myotis daubentonii (errata version published in 2021). IUCN Red List Threat. Species 2020, e.T85342710A195858793. [CrossRef]

33. Coroiu, I.; Juste, J.; Paunović, M. Myotis myotis. IUCN Red List Threat. Species 2016, e.T14133A22051759. [CrossRef]

34. Jiang, T.L.; Feng, J.; Csorba, G.; Bates, P. Myotis pilosus. IUCN Red List Threat. Species 2019, e.T14193A22062554. [CrossRef]

35. Feng, J.; Jiang, T.L. Myotis pequinius. IUCN Red List Threat. Species 2019, e.T14190A22066613. [CrossRef]

36. Fukui, D.; Sano, A.; Kruskop, S.V. Vespertilio sinensis. IUCN Red List Threat. Species 2019, e.T22949A22071812. [CrossRef]

37. Coroiu, I. Eptesicus nilssonii. IUCN Red List Threat. Species 2016, e.T7910A22116204. [CrossRef]

38. Csorba, G.; Hutson, A.M. Nyctalus noctula. IUCN Red List Threat. Species 2016, e.T14920A22015682. [CrossRef]

39. Godlevska, L.; Kruskop, S.V.; Gazaryan, S. Eptesicus serotinus (amended version of 2020 assessment). IUCN Red List Threat. Species 2021, e.T85199559A195834153. [CrossRef]

40. Gazaryan, S.; Bücs, S.; Çoraman, E. Miniopterus schreibersii (errata version published in 2021). IUCN Red List Threat. Species 2020, e.T81633057A195856522. [CrossRef]

41. Piraccini, R. Rhinolophus ferrumequinum. IUCN Red List Threat. Species 2016, e.T19517A21973253. [CrossRef]

42. Juste, J.; Alcaldé, J. Rhinolophus euryale. IUCN Red List Threat. Species 2016, e.T19516A21971185. [CrossRef] 
43. Juste, J.; Paunović, M. Myotis blythii. IUCN Red List Threat. Species 2016, e.T14124A22053297. [CrossRef]

44. Piraccini, R. Myotis emarginatus. IUCN Red List Threat. Species 2016, e.T14129A22051191. [CrossRef]

45. ESRI. ArcGIS Desktop: Release 10.8; Environmental Systems Research Institute: Redlands, CA, USA, 2021.

46. R Core Team. R: A Language and Environment for Statistical Computing; R Foundation for Statistical Computing: Vienna, Austria, 2021; Available online: http:/ / www.R-project.org/ (accessed on 31 March 2021).

47. Gouilh, M.A.; Puechmaille, S.J.; Gonzalez, J.P.; Teeling, E.; Kittayapong, P.; Manuguerra, J.C. SARS-Coronavirus ancestor's foot-prints in South-East Asian bat colonies and the refuge theory. Infect. Genet. Evol. 2011, 11, 1690-1702. [CrossRef]

48. Dominguez, S.R.; O'Shea, T.J.; Oko, L.M.; Holmes, K.V. Detection of group 1 coronavirus in bats in north America. Emerg. Infect. Dis. 2007, 13, e9. [CrossRef]

49. Kohl, C.; Nitsche, A.; Kurth, A. Update on Potentially Zoonotic Viruses of European Bats. Vaccines 2021, 9, 690. [CrossRef]

50. Dietz, C.; Kiefer, A. Bats of Britain and Europe; Bloomsbury Publishing: London, UK, 2016.

51. Ar Gouilh, M.; Puechmaille, S.J.; Diancourt, L.; Vandenbogaert, M.; Serra-Cobo, J.; Lopez Roïg, M.; Brown, P.; Moutou, F.; Caro, V.; Vabret, A.; et al. SARS-CoV related Betacoronavirus and diverse Alphacoronavirus members found in western old-world. Virology 2018, 517, 88-97. [CrossRef] [PubMed]

52. Reusken, C.B.; Lina, P.H.; Pielaat, A.; de Vries, A.; Dam-Deisz, C.; Adema, J.; Drexler, J.F.; Drosten, C.; Kooi, E.A. Circulation of group 2 coronaviruses in a bat species common to urban areas in Western Europe. Vector-Borne Zoonotic Dis. 2010, 10, 785-791. [CrossRef] [PubMed]

53. De Benedictis, P.; Marciano, S.; Scaravelli, D.; Priori, P.; Zecchin, B.; Capua, I.; Monne, I.; Cattoli, G. Alpha and lineage C BetaCoV infections in Italian bats. Virus Genes 2014, 48, 366-371. [CrossRef] [PubMed]

54. Falcón, A.; Vázquez-Morón, S.; Casas, I.; Aznar, C.; Ruiz, G.; Pozo, F.; Perez-Brena, P.; Juste, J.; Ibánez, C.; Garin, I.; et al. Detection of alpha and betacoronaviruses in multiple Iberian bat species. Arch. Virol. 2011, 156, 1883-1890. [CrossRef]

55. Mendenhall, I.H.; Kerimbayev, A.A.; Strochkov, V.M.; Sultankulova, K.T.; Kopeyev, S.K.; Su, Y.C.F.; Smith, G.J.D.; Orynbayev, M.B. Discovery and Characterization of Novel Bat Coronavirus Lineages from Kazakhstan. Viruses 2019, 11, 356. [CrossRef] [PubMed]

56. Ge, X.Y.; Wang, N.; Zhang, W.; Hu, B.; Li, B.; Zhang, Y.Z.; Zhou, J.H.; Luo, C.M.; Yang, X.L.; Wu, L.J.; et al. Coexistence of multiple coronaviruses in several bat colonies in an abandoned mineshaft. Virol. Sin. 2016, 31, 31-40. [CrossRef]

57. Chen, L.; Liu, W.; Zhang, Q.; Xu, K.; Ye, G.; Wu, W.; Sun, Z.; Liu, F.; Wu, K.; Zhong, B.; et al. RNA based mNGS approach identifies a novel human coronavirus from two individual pneumonia cases in 2019 Wuhan outbreak. Emerg. Microbes Infect. 2020, 9, 313-319. [CrossRef]

58. Lau, S.K.; Woo, P.C.; Li, K.S.; Huang, Y.; Tsoi, H.W.; Wong, B.H.; Wong, S.S.; Leung, S.Y.; Chan, K.H.; Yuen, K.Y. Severe acute respiratory syndrome coronavirus-like virus in Chinese horseshoe bats. Proc. Natl. Acad. Sci. USA 2005, 102, 14040-14045. [CrossRef]

59. Kim, H.K.; Yoon, S.W.; Kim, D.J.; Koo, B.S.; Noh, J.Y.; Kim, J.H.; Choi, Y.G.; Na, W.; Chang, K.T.; Song, D.; et al. Detection of Severe Acute Respiratory Syndrome-Like, Middle East Respiratory Syndrome-Like Bat Coronaviruses and Group H Rotavirus in Faeces of Korean Bats. Transbound. Emerg. Dis. 2016, 63, 365-372. [CrossRef] [PubMed]

60. Wu, Z.; Yang, L.; Ren, X.; He, G.; Zhang, J.; Yang, J.; Qian, Z.; Dong, J.; Sun, L.; Zhu, Y.; et al. Deciphering the bat virome catalog to better understand the ecological diversity of bat viruses and the bat origin of emerging infectious diseases. ISME J. 2016, 10, 609-620. [CrossRef]

61. Xu, L.; Zhang, F.; Yang, W.; Jiang, T.; Lu, G.; He, B.; Li, X.; Hu, T.; Chen, G.; Feng, Y.; et al. Detection and characterization of diverse alpha- and betacoronaviruses from bats in China. Virol. Sin. 2016, 31, 69-77. [CrossRef]

62. Tang, X.C.; Zhang, J.X.; Zhang, S.Y.; Wang, P.; Fan, X.H.; Li, L.F.; Li, G.; Dong, B.Q.; Liu, W.; Cheung, C.L.; et al. Prevalence and genetic diversity of coronaviruses in bats from China. J. Virol. 2006, 80, 7481-7490. [CrossRef] [PubMed]

63. Jevšnik, M.; Uršič, T.; Zigon, N.; Lusa, L.; Krivec, U.; Petrovec, M. Coronavirus infections in hospitalized pediatric patients with acute respiratory tract disease. BMC Infect. Dis. 2012, 12, 365. [CrossRef] [PubMed]

64. Zhou, P.; Yang, X.L.; Wang, X.G.; Hu, B.; Zhang, L.; Zhang, W.; Si, H.R.; Zhu, Y.; Li, B.; Huang, C.L.; et al. A pneumonia outbreak associated with a new coronavirus of probable bat origin. Nature 2020, 579, 270-273. [CrossRef] 